\title{
(305) $\mathrm{SrTiO}_{3}$ as substrate for coherently tilted epitaxial $\mathrm{YBa}_{2} \mathrm{Cu}_{3} \mathrm{O}_{x}$ thin films
}

\author{
W. A. M. Aarnink, E. M. C. M. Reuvekamp, M. A. J. Verhoeven, M. V. Pedyash, G. J. \\ Gerritsma, A. van Silfhout, and H. Rogalla \\ University of Twente, P. O. Box 217, 7500 AE Enschede, The Netherlands \\ T. W. Ryan \\ Philips Analytical, Lelyweg 1, 7602 EA Almelo, The Netherlands
}

(Received 12 February 1992; accepted for publication 20 May 1992)

\begin{abstract}
High $T_{c}$ superconducting $\mathrm{YBa}_{2} \mathrm{Cu}_{3} \mathrm{O}_{x}$ (YBCO) thin films have been prepared on (305) $\mathrm{SrTiO}_{3}$ (STO) substrates. X-ray diffraction analysis and Rutherford backscattering experiments reveal that the $c$-axis of the layers is directed along the [001] STO axis. Bragg reflection measurements from YBCO lattice planes with high $h, k$, and $l$ indices confirm that the film growth is epitaxial and almost single domain. For the critical current density $j_{c}(77 \mathrm{~K})$ values of $2 \times 10^{6}$ and $1 \times 10^{3} \mathrm{~A} / \mathrm{cm}^{2}$ have been found in the [010] and [50 $\left.\overline{1}\right] \mathrm{YBCO}$ directions, respectively.
\end{abstract}

The question of the superconducting properties anisotropy in the case of the high $T_{c}$ superconductors (HTS) is of great importance now. Recent experiments show strong anisotropy in coherence length $\xi$, critical current density $j_{c}$ and resistivity $\rho$ above the critical transition temperature $T_{c}^{4}$ between the directions parallel and perpendicular to the $\mathrm{CuO}_{2}$ planes in the $\mathrm{YBa}_{2} \mathrm{Cu}_{3} \mathrm{O}_{x}$ (YBCO). The origin of such behavior may be in the quasi-two-dimensional nature of high $T_{c}$ superconductivity. ${ }^{5}$

Measurements of the anisotropy of the transport properties on YBCO single crystals are difficult because of the well pronounced layered nature that inhibits the preparation of monocrystalline structures with dimensions in the $c$-direction large enough for $j_{c}$ measurements. Requirements of YBCO thin films are also exceptionally high. One should use epitaxial films which are single domain, properly oriented and free from twins.

The aim of this work is to study the possibility of the growth of coherently tilted epitaxial YBCO thin films on specially oriented (305) $\mathrm{SrTiO}_{3}$ (STO) substrates and apply these for the study of the anisotropy in the YBCO transport properties. Jia et al. showed that YBCO can cover steps on the (001) STO substrates. If the angle $\alpha$ between the normal to the (macroscopic) step surface and the (001) STO direction is below $\approx 40^{\circ}$, the YBCO on the step is $c$-axis oriented. ${ }^{6}$ The surface of our (305) STO substrates is similar to that of a step with $\alpha=30.96^{\circ}$. Therefore, we expect YBCO to grow on (305) STO substrates with the $c$-axis tilted with an angle of $31^{\circ}$ relative to the substrate surface normal, and directed along the [001] STO axis, see Fig. 1. If epitaxial and single domain films can be made, they allow us to study the anisotropy in the electrical properties of YBCO thin films for the first time, then also anisotropic behavior of many other physical properties of YBCO can be studied on these films.

We have studied single and double layers of $\mathrm{YBCO}$ and $\mathrm{PrBa}_{2} \mathrm{Cu}_{3} \mathrm{O}_{x}$ (PBCO), grown on (305) STO (Akzo International Research SCT) using off-axis if magnetron sputtering as a deposition technique. The deposition setup and typical sputter parameters are described elsewhere. ${ }^{7}$
X-ray diffraction analysis (XRD) and $2 \mathrm{MeV}{ }^{4} \mathrm{He}^{+}$ Rutherford backscattering spectrometry (RBS) were used to determine the orientation of the (double) layers. The XRD experiments were performed on a Philips Materials Research Diffractometer (MRD) equipped with parallel beam $x$-ray optics. ${ }^{8}$ The RBS data were analyzed using the RUMP computer code. ${ }^{9}$

Superconducting properties have been derived from the critical temperature $T_{c, \text { zero }}$, resistivity $\rho$, and critical current density $j_{c}$ measurements. We have prepared YBCO strips with different angles $\varphi$ between the direction of the strip and the [010] STO axis. They enable us to study the anisotropy in $j_{c}$ in our coherently tilted YBCO films. For $\varphi=0^{\circ}$, the measuring current can flow along the $a-b$ planes of the YBCO along the [010] direction and the critical current density $j_{c, \|}$, parallel to the $\mathrm{CuO}_{2}$ planes, can be determined. For $\varphi=90^{\circ}$, the current is forced to flow along the [501] direction of the YBCO. The critical current density measured along this direction will be near $j_{c, L}$, the critical current density perpendicular to the $\mathrm{CuO}_{2}$ planes.

The MRD is designed to allow one- or twodimensional scanning of any region of reciprocal space. $A$ $2 \theta / \omega$ scan directed along the STO $c$-axis, the STO [001] direction in Fig. 1, revealed strong $(0010),\left(\begin{array}{lll}0 & 0 & 11\end{array}\right)$, and $(0013)$ peaks from the superconductor (see Fig. 2). The (009) and (0 0 12) reflections from the YBCO coincided with the (003) and (004) reflections of the STO, respectively. These results confirm that the $c$-axis of the YBCO is tilted over $31^{\circ}$ when referred to the surface normal and parallel to the [001] STO direction.

To investigate the single-crystal nature of the YBCO film, reciprocal space maps of a series of Bragg reflections with high $h, k$, and $l$ indices have been measured. ${ }^{8}$ Figure 3 shows a map of the (038)-(308) pair of Bragg reflections. The measured difference in $2 \theta$ angle of $1.34^{\circ}$ corresponds exactly to the calculated value. The relative intensities of the (038) and (308) peaks indicate that the film is almost single domain with the $b$-axis of the YBCO lying in the (305) STO surface plane (see Fig. 1). The full width at half maximum (FWHM) of the rocking curve of the 


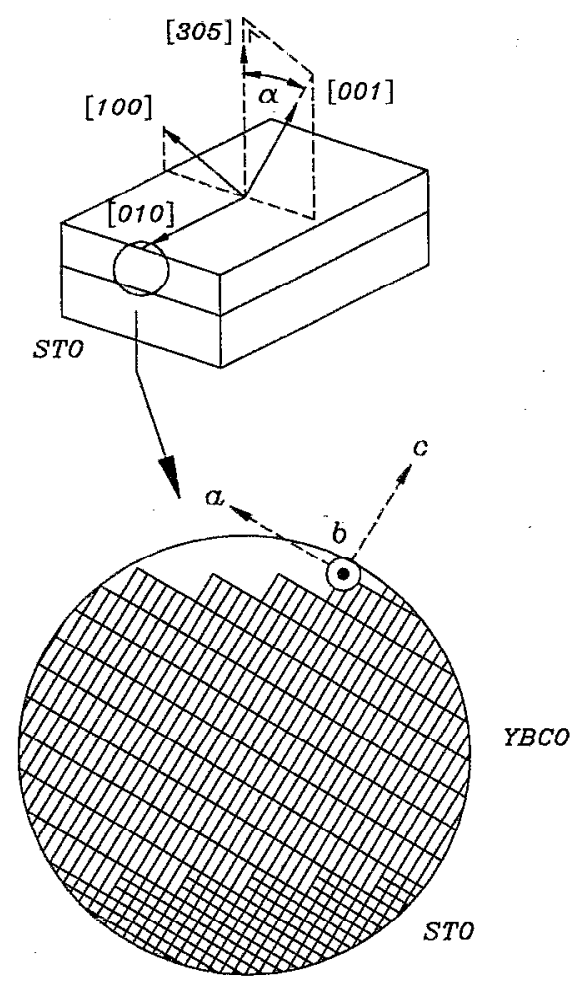

FIG. 1. Schematic, idealized view of a coherently tilted epitaxial YBCO thin film on a (305) STO substrate. The $c$-axis of the YBCO is tilted over $\alpha \approx 31^{\circ}$ when referred to the (macroscopic) surface normal.

(308) Bragg reflection is less then $1^{\circ}$, as can be seen in Fig. 3. A map of the (025)-(205) pair of reflections gave similar results. The present $\mathrm{x}$-ray diffraction studies indicate that the film is almost entirely single domain.

We have performed resistivity measurements on two sets of 5 YBCO strips with $\varphi=0^{\circ}, 22.5^{\circ}, 45^{\circ}, 67.5^{\circ}$, and $90^{\circ}$ as a function of temperature $T$. The strips have a length of 200 and $100 \mu \mathrm{m}$ and their widths equal 40 and $10 \mu \mathrm{m}$, respectively. The YBCO film thickness is $74 \mathrm{~nm}$. All the strips with a width of $40 \mu \mathrm{m}$ showed a $T_{c, \text { zero }}$ of $88 \mathrm{~K}$. The

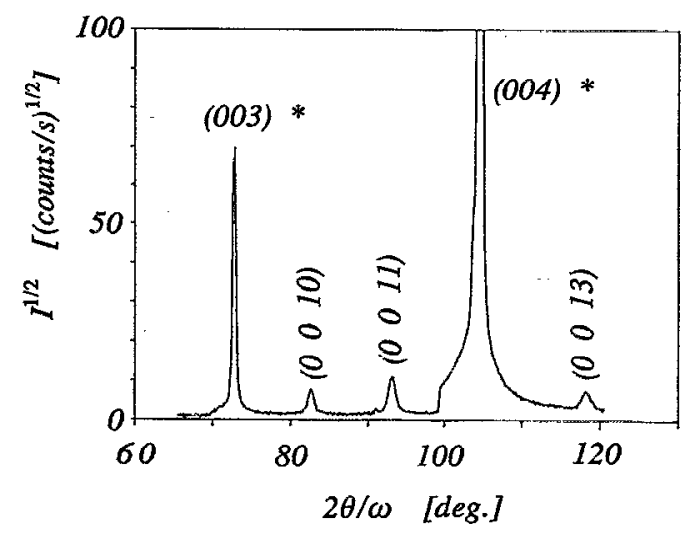

FIG. 2. $2 \theta / \omega \mathrm{XRD}$ scan along the STO $c$-axis, confirming that the $c$-axis of the $Y B C O$ is tilted over $31^{\circ}$ when referred to the surface normal and parallel to the [001] STO direction. STO substrate peaks are labeled with "**". Reflections from the YBCO are labeled with their corresponding indices. Note that the root of the X-ray intensity $I$ has been plotted as a function of $2 \theta / \omega$.

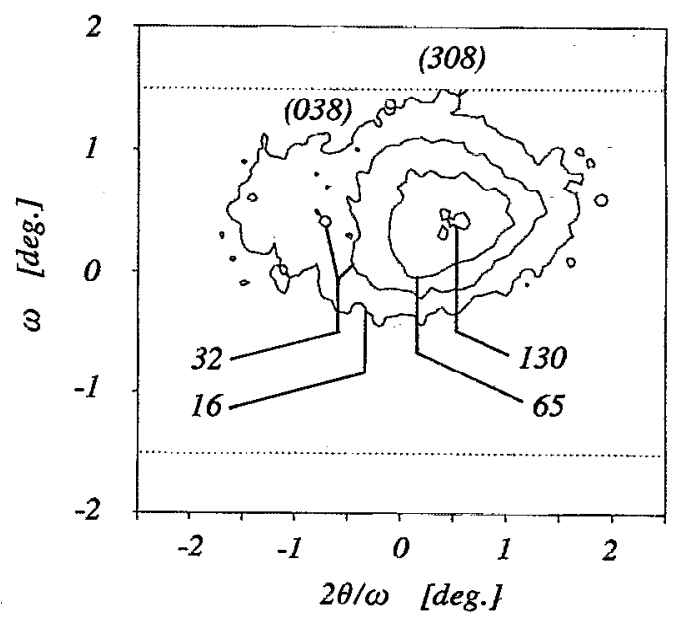

FIG. 3. Measurements of the (038)-(308) Bragg reflections pair of the YBCO film on a (305) STO/YBCO sample. Intensities of the different levels are indicated.

$T_{c, \text { zero }}$ of the strips with a width of $10 \mu \mathrm{m}$ decreased from 88 to $84 \mathrm{~K}$ upon increasing $\varphi$. However, $T_{c \text {,onset }}$ remained unchanged. In Fig. $4, \rho$ measured at $100 \mathrm{~K}$ on the YBCO strips with different $\varphi$ is shown. We see a strong anisotropy in $\rho(100 \mathrm{~K})$, comparable to the results of Penney et al. ${ }^{4}$ Also the resistivity ratio $\rho(300) / \rho(100)$ (RRR) depends on the width $w$ of the YBCO strip and on $q$, see Table $I$.

We also have measured $j_{c}$ at $77 \mathrm{~K}$ on the two sets of YBCO strips with different $w$ and orientation $\varphi$. In these measurements we used a criterion of $0.01 \mathrm{mV} / \mathrm{cm}$. A very strong anisotropy can be observed (see Fig. 4). We have found values of $2 \times 10^{6}$ and $1 \times 10^{3} \mathrm{~A} / \mathrm{cm}^{2}$ for $j_{c}(77 \mathrm{~K})$ in the [010] and [501] YBCO directions, respectively. Our results indicate a much larger anisotropy in $j_{c}$ than rcsults of Dinger et $a l^{2}$ and Crabtree $e t a l^{3}$

Application in microelectronics requires that YBCO can be used in multilayers, e.g., in combination with PBCO. In Fig. 5, results of RBS experiments on a (305) $\mathrm{STO} / \mathrm{YBCO} / \mathrm{PBCO}$ sample are given. A random spectrum

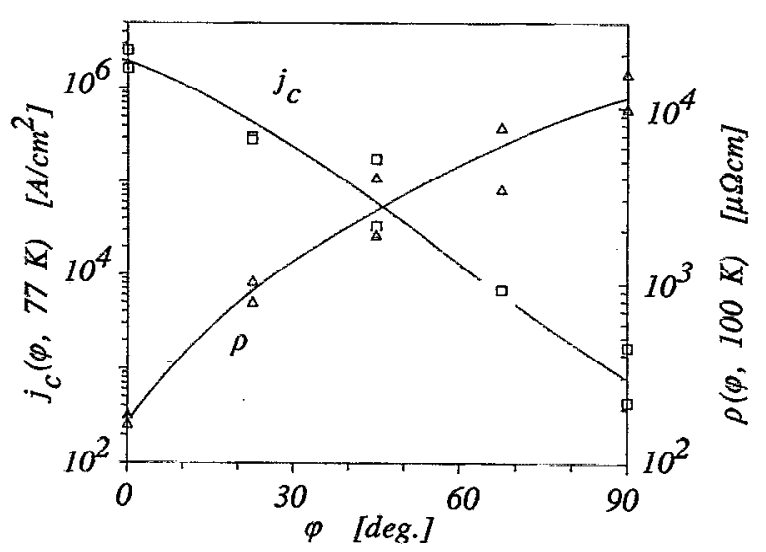

FIG. 4. Critical current density $j_{c}$ at $77 \mathrm{bK}$ and resistivity $\rho$ at $100 \mathrm{~K}$ measured on two sets of 5 YBCO strips on (305) STO as a function of the angle $\varphi$ between the direction of the strip and the [010] STO direction. $j_{c}$ measurements are indicated by $\square$, the $\rho$ measurements by $\Delta$. The solid lines are guides to the eye. 
TABLE I. $\operatorname{RRR}(w, \varphi)$ as a function of the width $w$ and the orientation $\varphi$ of an YBCO strip on (305) STO.

\begin{tabular}{cccccc}
\hline$\varphi[$ deg.] & 0 & 22.5 & 45 & 67.5 & 90 \\
\hline $\operatorname{RRR}(40 \mu \mathrm{m}, \varphi)$ & 2.40 & 1.57 & 1.33 & 1.31 & 1.28 \\
$\operatorname{RRR}(10 \mu \mathrm{m}, \varphi)$ & 2.28 & 1.24 & 1.13 & 1.01 & 0.56 \\
\hline
\end{tabular}

and a channeling spectrum, taken with the HE ion beam aligned with the [001] axis of the (305) STO substrate, are shown. For the YBCO bottom layer, a minimum backscattered yield $\chi_{\min }$ of about $\approx 30 \%$ is found. For the top PBCO layer, $\chi_{\min }$ is only $6 \%$. These low $\chi_{\min }$ values indicate that the $\mathrm{YBCO} / \mathrm{PBCO}$ double layer is well oriented, especially the PBCO top layer. In simulating the RBS spectrum in Fig. 5, we found for the YBCO and PBCO layer

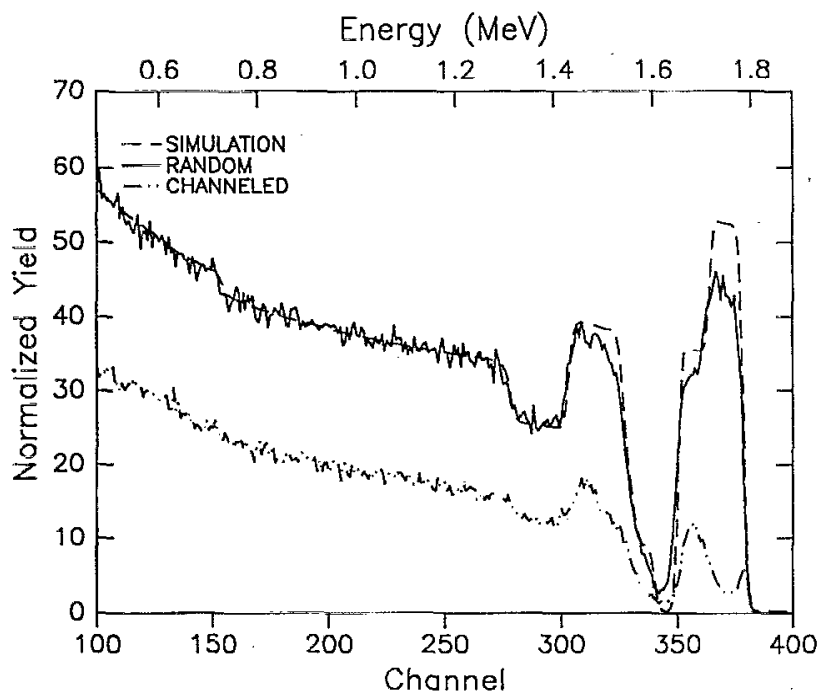

FIG. 5. RBS experiments on a (305) STO/YBCO/PBCO sample. The sample was mounted with a tilt of $31^{\circ}$ in the channeling experiments, so the He ion beam was parallel to the [001] STO direction. thicknesses values of 60 and $100 \mathrm{~nm}$, respectively. No significant interface reactions can be observed.

As a conclusion, epitaxial high $T_{c}$ superconducting single and double layers of YBCO and PBCO have been prepared on (305) STO substrates. X-ray diffraction analysis and Rutherford backscattering experiments reveal that the $c$-axis of the layers is directed along the [001] axis of the STO. Bragg reflection measurements from YBCO lattice planes with high $h, k$, and $l$ indices confirm that the film growth is epitaxial, and almost single domain. About $90 \%$ of the YBCO thin film has its $b$-axis parallel to the (305) STO surface, whereas the remaining part of the film has the $a$-axis parallel to this direction. For the critical current density $j_{c}(77 \mathrm{~K})$ values of $2 \times 10^{6}$ and $1 \times 10^{3} \mathrm{~A} / \mathrm{cm}^{2}$ have been found in the [010] and [501] directions of the YBCO, respectively.

This work is part of the research program of the "Stichting voor Fundamenteel Onderzoek der Materie (FOM)", which financially supported by the "Nederlandse organisatie voor Wetenschappelijk Onderzoek (NWO)", and was made possible by support of the "FOM-Instituut" voor Atoom en Molecuul Fysica (AMOLF)", Amsterdam and the "Centrum voor Materialen Onderzoek (CMO)", Enschede. D.H.A. Blank is thankfully acknowledged for his assistance in the preparation of the samples for the critical current density measurements.

${ }^{1}$ B. Batlogg, T. T. M. Palstra, L. F. Schneemeyer, R. B. van Dover, and R. J. Cava, Physica C 153-155, 1062 (1988).

${ }^{2}$ T. R. Dinger, T. K. Worthington, W. J. Gallagher, and R. L. Sandstrom, Phys. Rev, Lett. 58, 2687 (1987).

${ }^{3}$ G. W. Crabtree, J. Z. Liu, A. Umezawa, W. K. Kwok, C. H. Sowers, S. K. Malik, B. W. Veal, D. J. Lam, M. B. Brodsky, and J. W. Downey, Phys. Rcv. B 36, 4021 (1987).

${ }^{4}$ T. Penney, S. von Molnár, D. Kaiser, F. Holtzberg, and A. W. Kleinsasser, Phys. Rev. B 38, 2918 (1988).

${ }^{5}$ M. Tachicki and S. Takahashi, Physica B 169, 121 (1991).

${ }^{6}$ C. L. Jia, B. Kabins, K. Urban, K. Herrman, G. J. Cui, J. Schubert, W. Zander, A. I. Braginski, and C. Heiden, Physica C 175, 545 (1991).

${ }^{7}$ J. Gao, B. Häuser, and H. Rogalla, J. Appl. Phys. 67, 2512 (1990).

${ }^{8}$ E. Houtman, T. W. Ryan, B. David, and V. Doormann, in Advances in $X$-ray Analysis (in press).

${ }^{9}$ L. R. Doolittle, Nucl. Instrum. Methods B 9, 344 (1985). 